\title{
5. Kongress der Stiftung für Naturheilkunde und Erfahrungsmedizin: «Heilung findet zuhause statt - Lebensthemen»
}

Die Stiftung für Naturheilkunde und Erfahrungsmedizin bezweckt unter anderem die Förderung in folgenden Schwerpunkten [1]:

- Die Förderung der Forschung und Lehre im Bereich Naturheilkunde und Erfahrungsmedizin.

- Die Förderung ausgewählter Aktivitäten im Rahmen der komplementären modernen Medizin.

- Die Durchführung und Unterstützung von Forschungstätigkeiten auf dem Gebiet der Naturheilkunde und Erfahrungsmedizin.

- Den Aufbau einer Dokumentation über bestehende und neue Therapien und Heilmittel im Bereich der Naturheilkunde und Erfahrungsmedizin.

- Das Anbieten von medizinischen und psychologischen Grundausbildungen auf selbsttragender Basis sowie die Zusammenarbeit mit bestehenden Ausbildungsinstitutionen.

- Die Überprüfung und Klassifizierung bestehender Schulungen und Abschlussdiplome im Bereich Naturheilkunde und Erfahrungsmedizin.

- Die publizistische Förderung der Naturheilkunde und Erfahrungsmedizin durch Vortragsveranstaltungen, Pressepublikationen, Broschüren, Gesundheitstage etc.

«Lebensthemen» sind Themen, die das ganze Leben umfassen, es gestalten und verändern. Sie können einen einzelnen Lebensabschnitt prägen oder ein ganzes Leben andauern, sich wan-
Am 3. und 4. Oktober 2014 fand in Solothurn zum 5. Mal der Kongress der Stiftung für Naturheilkunde und Erfahrungsmedizin (SNE) statt. Der Kongress mit dem Titel «Heilung findet zuhause statt - Lebensthemen» ist eine Aktivität der 1995 von der EGK-Gesundheitskasse gegründeten SNE.

deln oder immer gleich sein. Lebensthemen betreffen Fragen der Gesundheit sowie der Lebensgestaltung auf sozialer, spiritueller, ökologischer und ökonomischer Ebene und wirken auf die Selbstheilungskräfte sowie die Persönlichkeitsbildung und Reifung des Menschen.

\section{Referate und Workshops}

Bekannte, auf ihrem Gebiet ausgewiesene Experten boten den Teilnehmenden die Möglichkeit, sich kritisch mit den «Lebensthemen» auseinanderzusetzen.

Ein Forschungsschwerpunkt von Prof.Dr. Matthias Binswanger (Abb. 1), Professor für Volkswirtschaftslehre, liegt in der Erforschung des Zusammenhangs von Glück und Einkommen. So ging Prof. Binswanger in seinem Referat der Frage nach, «warum mehr Einkommen nicht glücklicher macht».

Es ist eine gut sichtbare Tatsache, dass die heutige Wettbewerbs- und Wachstumsgesellschaft die Menschen nicht glücklich macht. Die durchschnittliche Zufriedenheit der Menschen in entwickelten Ländern nimmt schon lange nicht mehr zu, obwohl die durchschnittlichen Einkommen sich

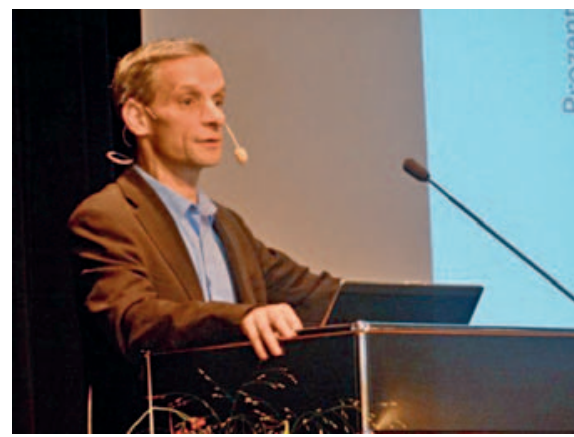

Abb. 1. Warum mehr Einkommen nicht glücklicher macht, erörterte Herr Prof. Dr. Matthias Binswanger.

mit dem Wirtschaftswachstum weiter erhöhen. Das belegen zahlreiche empirische Studien [1]. Prof. Binswanger sieht den Grund im sogenannten «Tretmühleneffekt»: Auch wenn man auf einer Tretmühle schneller läuft, bleibt man immer am selben Ort. Das menschliche Streben, durch mehr Einkommen und mehr Konsum glücklicher zu werden, kann demnach nicht funktionieren. Zwar werden die Menschen reicher und reicher; was aber das Empfinden von Glück und Zufriedenheit betrifft, so ändert sich nichts. Im Gegenteil: Die Hoffnung auf mehr Glück wird ständig enttäuscht.

Dr. med. Simon Feldhaus, Facharzt für Allgemeinmedizin, medizinischer Leiter Paramed, Baar, und Präsident

\begin{tabular}{ll}
\hline KARGER & @ 2014 S. Karger GmbH, Freiburg \\
& Accessible online at: \\
$\begin{array}{l}\text { Fax }+497614520714 \\
\text { Information@Karger.com } \\
\text { www.karger.com }\end{array}$ & www.karger.com/szg
\end{tabular}


SSAAMP (Swiss Society for Anti Aging Medicine and Prevention), betrachtete in seinem Referat die "Gesundheit aus regulationsmedizinischer Sicht». Im Sinne von Aristoteles' Aussage «Das Ganze ist mehr als die Summe seiner Teile» wird der Mensch als vernetztes System mit einem stetigen Austausch von Energie und Materie gesehen. Gesundheit ist damit ein Fliessgleichgewicht von seelischen, geistigen, körperlichen und energetischen Aspekten. In der Diagnostik und Therapie der Regulationsmedizin wird der Erkennung und Behandlung von Störfeldern, Toxikosen, Mangelzuständen oder anderen Regulationsblockaden darum höchste Priorität beigemessen.

Lothar Hirneise, Vorstand von Krebs21, ehemaliger Forschungsdirektor der National Foundation for Alternative Medicine in Washington D.C. und Autor, zeigte in seinem Referat «Erfolgreiche, ganzheitliche Krebstherapien» Konzepte aus der alternativen Krebsbehandlung.

Dr. med. Hans P. Ogal, Facharzt für Anästhesiologie, Schmerztherapie und Akupunktur und zudem Autor, sprach über chronische Schmerzerkrankungen mit dem Fokus «Schmerzen ganzheitlich verstehen und behandeln». Um die Entstehung einer Schmerzkrankheit zu verhindern, müssen die aktuelle Symptomatik und die tiefer liegenden Ursachen des Schmerzes gleichermassen betrachtet und behandelt werden. Während akute Schmerzen bekanntermassen lebenswichtige Warnsignale darstellen, können sich solche, die nicht rechtzeitig oder nur ungenügend behandelt werden, zu einer Schmerzkrankheit entwickeln. Eine ganzheitlich ausgerichtete Schmerztherapie ist dann erfolgreich, wenn unter der Vielzahl schul- und komplementärmedizinischer Verfahren das individuell wirkungsvollste Therapiekonzept gefunden wird.

Dr. med. Eckhart Pape, praktischer Arzt FMH für Biologische Medizin, ging in seinem Vortrag auf die Pro-

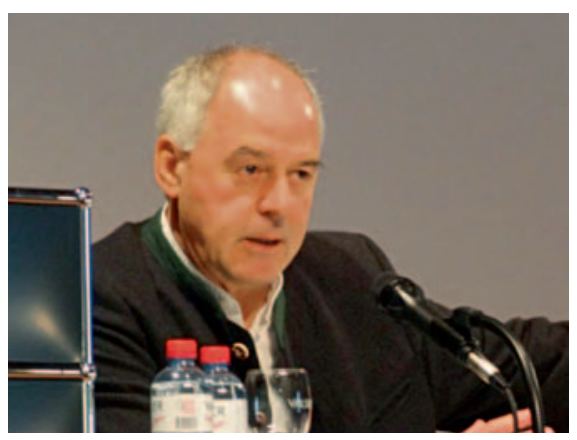

Abb. 2. Herr Prof. Dr. phil. Wilhelm Schmid präsentierte seine Gedanken und Erkenntnisse zu «Gesundheit und Lebenskunst».

blematik der Suchterkrankungen («Sucht: Siechtum oder Suche») ein. Wird der Konsum psychotroper Substanzen als Spiegel sowohl der Gesellschaft als auch des Individuums gesehen, werden Suchttendenzen zu tiefgreifenden Lebensthemen - und zwar für den direkt Betroffenen wie auch für sein ganzes Umfeld ('Co-Abhängigkeit').

Prof. Dr. phil. Wilhelm Schmid (Abb. 2), freier Philosoph, war etliche Jahre am Schweizer Spital in Affoltern als philosophischer Seelsorger tätig. In seinen philosophischen Überlegungen zum Thema «Gesundheit und Lebenskunst» forderte er die Zuhörenden auf, ein oberflächliches Verständnis von Gesundheit kritisch zu hinterfragen. Dass es die widersprüchlichen Phänomene «Gesundheit» und «Krankheit» überhaupt gibt, zeigt gemäss Prof. Schmid das Bedürfnis des Lebens nach Polarität. Störungen im Leben sind nötig, da Störungen die Gesundheit stärken - was das heutige Wissen über das Immunsystem und seine Leistungsfähigkeit bestätigt. Prof. Schmid stellte damit die Vorstellung, der Mensch sollte immer gesund sein, infrage. Welch grosse Anstrengungen hat die Menschheit doch unternommen, um Krankheiten auszumerzen. Warum hält sich dennoch die Polarität zwischen Gesundheit und Krankheit? Philosophisch gesehen gehört Krankheit untrennbar zum Menschsein, da der Mensch die Gegensätze zum Leben braucht. Heute ist die Lehre der Polarität vor allem aus dem asi- atischen Raum (Yin/Yang) bekannt. Doch auch in unserem Kulturkreis (Aristoteles, Heraklit, Nietzsche usw.) ist die Vorstellung bekannt, dass Gegensätze zusammengehören und erst die Polarität das Leben ermöglicht. So gesehen ist Krankheit eine Aufforderung, das Leben (um-)zugestalten, die Persönlichkeit zu formen, notwendige (Not wendende) Schritte zu machen und damit die ureigenen Lebensthemen zu leben.

Tatjana Strobel, Physiognomie-Expertin, zeigte in ihrem Referat «Entschlüsseln Sie das Mysterium Mensch» auf, wie sehr Worte täuschen können. Der Mensch kommuniziert nur 20\% über den Inhalt seiner Worte. 80\% verrät der Mensch über seine Körpersprache, seine Gesten, die Mimik und die Art seiner Sprache. Da jeder Gedanke eine Gehirnaktivität ist, die eine Muskelaktivität erzeugt und sich in Bewegungen und in der Ausstrahlung zeigt, erkennt ein aufmerksamer Mensch bald, wie es seinem Gegenüber gerade geht. Lange bevor ein Mensch etwas sagt, hat er seine Gefühle bereits zum Ausdruck gebracht. Eine ganzheitliche Heilkunst muss diese Menschenkenntnis miteinschliessen, um den Menschen wirklich umfassend und individuell wahrnehmen, behandeln und begleiten zu können.

Dr. med. dent. Urs Weilenmann, Zahnmediziner SSO (Schweizerische Zahnärzte-Gesellschaft) und ganzheitlicher Zahnmediziner SGZM (Schweizerische Gesellschaft für ganzheitliche Zahnmedizin), sprach über «das Phänomen Störfeld aus zahnärztlicher Sicht». Er zeigte auf, dass sich bei komplementärmedizinischen Therapien oft Verbesserungen von Pathologien ergeben, wenn Belastungen des Organismus reduziert werden. Diese Pathologien werden jedoch mit schulmedizinischer Diagnostik meist nicht entdeckt. Für wissenschaftliche Erklärungen müssen die Chaostheorie und die Quantenphysik hinzugezogen und diskutiert werden.

Beat Hampe, Fachmann für Astronomie, Anatomie und Psychologie, er- 
örterte in seinem Workshop zum Thema «Welcher Beziehungstyp bin ich?» die Dynamik der grundsätzlichen Beziehungstypen und deren individuelle Lebensausprägung.

Eva Lackner, Heilpraktikerin aus Deutschland, zeigte anhand der pythagoräischen Numerologie auf, «wie Zahlen das Leben bestimmen bzw. wie der Mensch mit Zahlen sein Leben bestimmt».
Die quantitative Ebene der Zahlen ist in der heutigen Gesellschaft allen bekannt. Dass Zahlen nicht nur zum Zählen da sind, sondern dass sie auch eine qualitative Dimension haben, ist hingegen weniger präsent. Basierend auf der pythagoräischen Numerologie wird die Bedeutung der Geometrie für das menschliche Leben sichtbar und ermöglicht ein tieferes Verständnis der Lebensthemen.
Fazit

Das spannende Symposium zeigte die verschiedenen Facetten von Lebensthemen auf, die eine ganzheitliche Menschenbetrachtung und Heilkunst mit sich bringt.

\section{Literatur}

1 www.stiftung-sne.ch. 